EPJ Web of Conferences 66, 05012 (2014)

DOI: $10.1051 /$ epjconf/ 20146605012

(C) Owned by the authors, published by EDP Sciences, 2014

\title{
High-Precision Half-life Measurements for the Superallowed $\beta^{+}$ Emitter ${ }^{14} \mathrm{O}$
}

\author{
A. T. Laffoley1,a, C. E. Svensson ${ }^{1}$, C. Andreoiu², R. A. E. Austin ${ }^{3}$, G. C. Ball ${ }^{4}$, B. Blank ${ }^{5}$, \\ H. Bouzomita ${ }^{6}$, D. S. Cross ${ }^{2}$, A. Diaz Varela ${ }^{1}$, R. Dunlop ${ }^{1}$, P. Finlay ${ }^{1}$, A. B. Garnsworthy ${ }^{4}$, \\ P. E. Garrett ${ }^{1}$, J. Giovinazzo ${ }^{5}$, G. F. Grinyer ${ }^{6}$, G. Hackman ${ }^{4}$, B. Hadinia ${ }^{1}$, D. S. Jamieson ${ }^{1}$, \\ S. Ketelhut ${ }^{4}$, K. G. Leach ${ }^{1}$, J. R. Leslie ${ }^{7}$, E. R. Tardiff ${ }^{4}$, J. C. Thomas ${ }^{6}$, and C. Unsworth ${ }^{4}$ \\ ${ }^{1}$ Department of Physics, University of Guelph, Guelph, Ontario N1G 2W1, Canada \\ ${ }^{2}$ Department of Chemistry, Simon Fraser University, Burnaby, British Columbia V5A 1S6, Canada \\ ${ }^{3}$ Department of Astronomy \& Physics, Saint Mary's University, Halifax, Nova Scotia B3H 3C3, Canada \\ ${ }^{4}$ TRIUMF, 4004 Wesbrook Mall, Vancouver, British Columbia V6T 2A3, Canada \\ ${ }^{5}$ Centre d'Etudes Nucléaires de Bordeaux Gradignan-Université Bordeaux 1-UMR 5797 CNRS/IN2P3, \\ Chemin du Solarium, BP 120, F-33175 Gradignan Cedex, France \\ ${ }^{6}$ GANIL, CEA/DSM-CNRS/IN2P3, Bvd Henri Becquerel, 14076 Caen, France \\ ${ }^{7}$ Department of Physics, Queen's University, Kingston, Ontario K7L 3N6, Canada
}

\begin{abstract}
The half-life of ${ }^{14} \mathrm{O}$, a superallowed Fermi $\beta^{+}$emitter, has been determined via simultaneous $\gamma$ and $\beta$ counting experiments at TRIUMF's Isotope Separator and Accelerator facility. Following the implantation of ${ }^{14} \mathrm{O}$ samples at the center of the $8 \pi$ spectrometer, a $\gamma$ counting measurement was performed by detecting the $2313 \mathrm{keV} \gamma$ rays emitted from the first excited state of the daughter ${ }^{14} \mathrm{~N}$ using 20 high-purity germanium (HPGe) detectors. A simultaneous $\beta$ counting experiment was performed using a fast plastic scintillator positioned directly behind the implantation site. The results, $T_{1 / 2}(\gamma)=70.632 \pm 0.094 \mathrm{~s}$ and $T_{1 / 2}(\beta)=70.610 \pm 0.030 \mathrm{~s}$, are consistent with one another and, together with eight previous measurements, establish a new average for the ${ }^{14} \mathrm{O}$ half-life of $T_{1 / 2}=70.619 \pm 0.011 \mathrm{~s}$ with a reduced $\chi^{2}$ of 0.99 .
\end{abstract}

\section{Introduction}

High-precision measurements of the $\beta$ decay $f t$ values for superallowed Fermi $\beta$ transitions between nuclear analog states of spin $J^{\pi}=0^{+}$and isospin $T=1$ provide demanding, and fundamental, tests of the properties of the electroweak interaction by directly probing the weak vector current [1]. Measurements of these $f t$ values have been used to validate the conserved vector current (CVC) hypothesis to better than 2 parts in $10^{4}$, provide the most precise determination of $V_{u d}$, by far the most precisely measured element of the Cabibbo-Kobayashi-Maskawa (CKM) quark mixing matrix [2], and are used to constrain the presence of induced or fundamental scalar currents in $\beta$ decay.

The presence of a fundamental scalar interaction (or one induced by the vector current) would affect the corrected $f t$ values, denoted $\mathcal{F} t$, for superallowed $\beta$ emitters such that they would cease

a e-mail: alaffole@uoguelph.ca

This is an Open Access article distributed under the terms of the Creative Commons Attribution License 2.0, which permits unrestricted use, distribution, and reproduction in any medium, provided the original work is properly cited. 
to be constant as a function of $Z$. For maximally parity violating scalar interactions, the $\mathcal{F} t$ values would contain an additional term approximately proportional to the average inverse decay energy of the $\beta^{+}$transition, $\langle 1 / Q\rangle$. The largest deviations of the $\mathcal{F} t$ values from a constant would occur in the lightest of the superallowed $\beta^{+}$emitters, ${ }^{10} \mathrm{C}$ and ${ }^{14} \mathrm{O}$, for which the decay $Q$-values are the smallest. The $f t$ values for these isotopes are therefore most important for setting limits on the existence of scalar weak interactions. A weighted average of the eight previous precision measurements of the ${ }^{14} \mathrm{O}$ half-life [3-10] yields $70.620(12) \mathrm{s}$, a result that is precise to $\pm 0.019 \%$, but there is cause to question the accuracy of this average $T_{1 / 2}$ value. Based on the reduced $\chi^{2}$ value of 1.26 , these results form an inconsistent data set and prompt Hardy and Towner in their world survey of superallowed data [1] to apply a scale factor of 1.2 to the statistical uncertainty of the ${ }^{14} \mathrm{O}$ half-life and adopt a value of $70.620 \pm 0.015 \mathrm{~s}$. The half-life of ${ }^{14} \mathrm{O}$ can be measured in two ways, either by directly counting the $\beta$ particles or measuring the $\gamma$ activity since, with a branching ratio of $99.4 \%,{ }^{14} \mathrm{O}$ decays to an excited state of its daughter ${ }^{14} \mathrm{~N}$ which then emits a $2313 \mathrm{keV} \gamma$-ray [11]. Grouping the previous measurements by detection method used reveals a discrepancy of $0.07 \%, T_{1 / 2}(\gamma)=70.598(17) \mathrm{s}$ and $T_{1 / 2}(\beta)=70.648(19) \mathrm{s}$, results that have a $\chi^{2} / v=3.85$ if considered as two independent techniques for the measurement of the same quantity. Choosing the average result from either of these methods would shift the ${ }^{14} \mathrm{O} f t$ value, one of the most precisely quoted of all of the superallowed decays, by $0.5 \sigma$. The same systematic effect appears to be present in previous half-life determinations for ${ }^{10} \mathrm{C}$ where the $f t$ value for this decay could potentially be modified by up to $0.6 \sigma$. These would have direct implications on the overall value deduced for $V_{u d}$, the test of CKM unitarity, and the present constraint on the existence of a fundamental or induced scalar interaction in the minimal electroweak Standard Model. It is this half-life discrepancy that has motivated the simultaneous high-precision $\beta$ and $\gamma$ counting ${ }^{14} \mathrm{O}$ half-life measurements reported here.

\section{Experiment}

The experiment was performed at TRIUMF's Isotope Separator and Accelerator (ISAC) facility. A beam of $500 \mathrm{MeV}$ protons impinged on a $\mathrm{SiC}$ target causing spallation reactions whose products diffused from the target and were ionized using a Forced Electron Beam Ion Arc Discharge (FEBIAD) ion source. A mass separator was used to select a beam of $A=26$ products, including the primary beam of carbon-monoxide $\left({ }^{12} \mathrm{C}^{14} \mathrm{O}\right)$ molecules, as well as contaminants of ${ }^{26} \mathrm{Na}$ and ${ }^{26} \mathrm{Al}^{m}$. The $30-\mathrm{keV}$ beam was delivered at an average rate of $1.2 \times 10^{4}{ }^{14} \mathrm{O} / \mathrm{s}, 2.4 \times 10^{6}{ }^{26} \mathrm{Na} / \mathrm{s}$, and $1.0 \times 10^{3}$ ${ }^{26} \mathrm{Al}^{m} / \mathrm{s}$ and was implanted, under vacuum, at the center of the $8 \pi$ spectrometer [12] into a movable mylar-backed aluminum tape-transport system [13] for 3 minutes prior to being deflected before the mass separator two floors below the experimental hall. The sample collected on the tape was then allowed to decay for $23 \mathrm{~min}$, or approximately twenty ${ }^{14} \mathrm{O}$ half-lives. This constituted a single run, at which time the tape was moved outside the array behind a thick lead wall to remove any long-lived contaminants $\left({ }^{26} \mathrm{Al}^{g}\right)$ from the array and the cycle was repeated; a total of 102 runs were collected during the experiment. It is known that the diffusion of gaseous samples can be an issue when using thin tape systems, but this effect has been studied with the $40-\mu \mathrm{m}$-thick layer of aluminum on a backing of mylar used at the $8 \pi$ which concluded that diffusion effects on this time scale are negligible [14]. The $8 \pi \gamma$-ray spectrometer, a spherically symmetric array of 20 HPGe detectors, and the Zero-Degree Scintillator (ZDS), a fast plastic scintillating detector positioned at zero degrees relative to the beam axis and directly behind the thin aluminized mylar tape on which the beam was implanted, were used to collect data. The non-extendible dead-time of the $8 \pi$ data acquisition system, as well as the shaping times of the HPGe spectroscopy amplifiers, were varied throughout the experiment in order to investigate possible systematic effects. Data were collected with combinations of three dead-time 

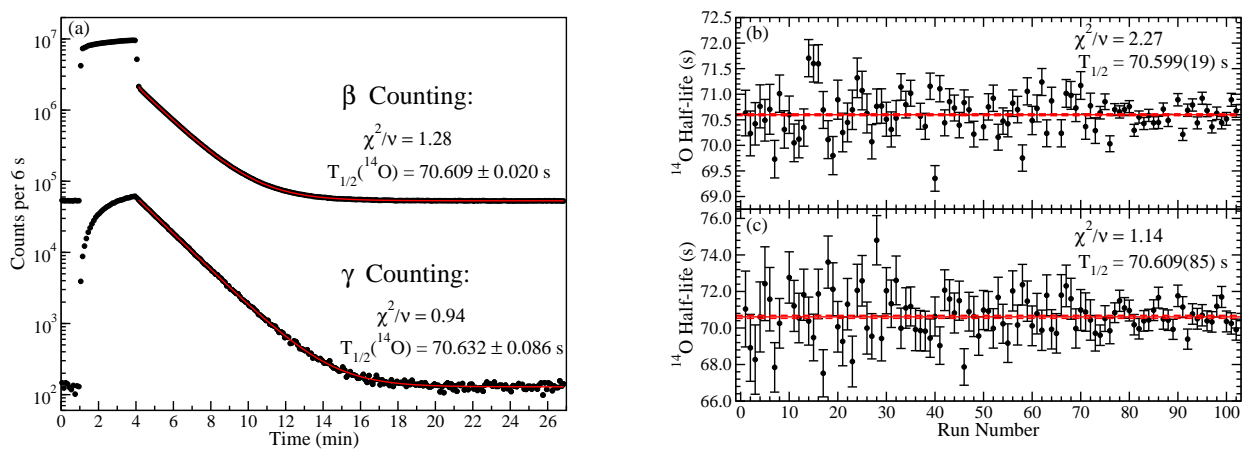

Figure 1. (a) Dead-time corrected activity curve of the summed (global) data from the $\beta$ (upper) and $\gamma$-ray (lower) counting experiments. The $\beta$ data were taken from the MCS data stream with $2 \mu$ s fixed dead-time applied. The $\gamma$ activity curve was also pile-up corrected and an appropriate $\gamma$-ray energy gate has been applied. The difference in relative contaminant intensities between the $\beta$ and $\gamma$ techniques can be clearly seen in the implantation growin behaviors between these two curves. (b) Half-life versus run number obtained through $\beta$ counting with the ZDS. A weighted average was performed on this set, and the result is shown by the solid line as well as the $1 \sigma$ uncertainties by dashed lines. (c) Half-life versus run number obtained from the $\gamma$-ray measurements.

settings (variable, fixed $30 \mu \mathrm{s}$, and fixed $50 \mu \mathrm{s}$ ) and three shaping times $(0.5 \mu \mathrm{s}, 1.0 \mu \mathrm{s}$, and $2.0 \mu \mathrm{s})$. The signals from $\beta$ particles detected in the ZDS were fanned out into 5 data streams and multiscaled into bins of 6 second duration using five independent channels of a VME-based multichannel scaler (MCS). Fixed and non-extendible dead-times, chosen to be much longer than the total series dead-time of the system, were applied to each MCS stream using LeCroy $222 \mathrm{~N}$ nonretriggerable gate-and-delay generators and were measured via the source-plus-pulser method [15] to be 1.9818(37), 5.0041(41), 10.0042(38), 20.0109(60), and 29.9906(86) $\mu$ s.

\section{Results and Discussion}

Following dead-time corrections [16, 17], the ZDS data were fit using a maximum-likelihood technique described in previous works [16-19]. The fitting routine included a component for the ${ }^{14} \mathrm{O}$ activity, the ${ }^{26} \mathrm{Na}$ activity, the ${ }^{26} \mathrm{Al}^{m}$ activity, and a constant background. The half-lives of both ${ }^{26} \mathrm{Na}$ and ${ }^{26} \mathrm{Al}^{m}$ were fixed at their precise values that were deduced in previous experiments $[17,18]$.

The $\gamma$-ray counting data from the $8 \pi$ spectrometer were dead-time and pile-up corrected $[14,16$, $21]$ before being fitted using the same routines as used with the $\beta$ data. Following the $\beta$ decay of ${ }^{26} \mathrm{Al}^{m}$, no known $\gamma$ radiation is produced [22] and the bremsstrahlung contribution at $2313 \mathrm{keV}$ from the ${ }^{26} \mathrm{Al}^{m}$ decay positrons, with $\beta$ end-point energy of $3211 \mathrm{keV}$, is also very small, so the activity due to ${ }^{26} \mathrm{Al}^{m}$ decay in the $2313 \mathrm{keV} \gamma$-ray window was entirely negligible. As a consistency check, the activity of ${ }^{26} \mathrm{Al}^{m}$ was also included in the fitting procedure as a free parameter but the activity derived from the fit was consistent with zero, and was treated as a fixed parameter in the analysis.

Figure 1(a) shows the summed decay curve in which all dead-time and pile-up corrected data from each of the runs were summed and a single global fit was performed. One can see the advantages of each detection method: a higher efficiency (almost 2 orders of magnitude) when directly counting the $\beta$ particles while there is a significant improvement in signal-to-noise ratio as well as decay selectivity (suppression of contaminant activity) in the $\gamma$-ray counting method. The results of the half-life deduced for each run in both the $\beta$ and $\gamma$ counting methods are highlighted in Figs. 1(b) and (c). 
A systematic investigation was performed in which the data were grouped according to similar experimental conditions, as detailed in Ref. [23]. The procedure adopted by the Particle Data Group was used to inflate the statistical error by the square root of the largest $\chi^{2}$ giving an estimate of the potential systematic error in each of the $\beta$ and $\gamma$-ray measurements.

\section{Conclusions}

Two simultaneous, but independent, high-precision half-life measurements for the superallowed Fermi $\beta^{+}$emitter ${ }^{14} \mathrm{O}$ were performed yielding results of $T_{1 / 2}(\gamma)=70.632 \pm 0.094 \mathrm{~s}$ and $T_{1 / 2}(\beta)=70.610 \pm$ $0.030 \mathrm{~s}$, respectively. This new $\beta$ counting measurement is in excellent agreement with the previous $\gamma$ counting results, whereas this new $\gamma$ counting measurement agrees with both groupings of previous data within $1 \sigma$. These results improve the precision of the world average for the ${ }^{14} \mathrm{O}$ half-life and yield a consistent world data set with $\chi^{2} / v=0.99$, thereby reducing the uncertainty in the world average adopted in Ref. [1] by $25 \%$ to $T_{1 / 2}\left({ }^{14} \mathrm{O}\right)=70.619 \pm 0.011 \mathrm{~s}$.

\section{Acknowledgements}

This work has been partially supported by the Natural Sciences and Engineering Research Council of Canada. TRIUMF receives federal funding via a contribution agreement with the National Research Council of Canada.

\section{References}

[1] J.C. Hardy and I.S. Towner, Phys. Rev. C 79, 055502 (2009).

[2] I.S. Towner and J.C. Hardy, Rep. Prog. Phys. 73, 046301 (2010).

[3] P.H. Barker, I.C. Barnett, G.J. Baxter, and A.P. Byrne, Phys. Rev. C 70, 024302 (2004).

[4] J.T. Burke et al., Phys. Rev. C 74, 025501 (2006).

[5] D.E. Alburger, Phys. Rev. C 5, 274 (1972).

[6] G.J. Clark et al., Nucl. Phys. A215, 429 (1973).

[7] G. Azuelos, J.E. Crawford, and J.E. Kitching, Phys. Rev. C 9, 1213 (1974).

[8] J.A. Becker et al., Nucl. Instrum. Methods 155, 211 (1978).

[9] D.H. Wilkinson, A. Gallmann, and D.E. Alburger, Phys. Rev. C 18, 401 (1978).

[10] M. Gaelens et al., Eur. Phys. J. A 11, 413 (2001).

[11] F. Ajzenberg-Selove, Nuclear Physics A 523, 1 (1991).

[12] C.E. Svensson et al., Nucl. Instrum. and Methods B 204, 660 (2003).

[13] G.C. Ball et al., J. Phys. G 31, S1491 (2005).

[14] G.F. Grinyer et al., Phys. Rev. C 76, 025503 (2007).

[15] A.P. Baerg, Metrologia 3, 131 (1965).

[16] G.F. Grinyer et al., Nucl. Instrum. Methods A 579, 1005 (2007).

[17] G.F. Grinyer et al., Phys. Rev. C 71, 044309 (2005).

[18] P. Finlay et al., Phys. Rev. Lett. 106, 032501 (2011).

[19] V.T. Koslowsky et al., Nucl. Instrum. Methods A401, 289 (1997).

[20] P.M. Endt, Nucl. Phys. A 633, 1 (1998).

[21] G.F. Grinyer et al., Phys. Rev. C 87, 045502 (2013).

[22] P. Finlay et al., Phys. Rev. C 85, 055501 (2012).

[23] A.T. Laffoley et al., Phys. Rev. C 88, 015501 (2013). 